\title{
DeVEloping AN AdAPTIVE CHANNEL MODELLING USING A GENETIC ALGORITHM TECHNIQUE TO ENHANCE AERIAL VEHICLE-TO- EVERYTHING WIRELESS COMMUNICATIONS
}

\author{
Faris. A. Almalki \\ Department of Computer Engineering, College of Computers and Information \\ Technology, Taif University, Kingdom of Saudi Arabia
}

\begin{abstract}
In this digital era, Internet of Everything (IoE) has a potential to bring out drastic changes to how we live today, where billions of people and devices require wireless connectivity. Where Unmanned Aerial Vehicles contribute positively in paving the way for IoE and Fifth Generation technologies, and tackle some of their comms challenges. Thus, this paper aims to provide an adaptive approach using a Genetic Algorithm (GA) technique by combining indoor and outdoor propagation models to enhance aerial vehicle-to-everything wireless connectivity. The proposed adaptive approach uses a GA multi-objective function that yield optimum values of UAV altitude, elevation angles, and type of building for indoor environment. The proposed GA optimization technique has met the demand of a typical dense-populated urban environment, as well as empowering the IoE with greater coverage footprint, high Quality of Service benchmark, and line-of-sight adaptability. The output results emphasized that the proposed adaptive approach using the GA technique can help in smart decision-making and selecting a proper setup and find the optimum parameters to provide seamless wireless connections from aerial vehicle-to-everything.
\end{abstract}

\section{KEYWORDS \\ Unmanned Aerial Vehicles, Internet of Everything, Channel Modelling, Propagation Model, Fifth Generation.}

\section{INTRODUCTION}

As reported in The Economist magazine, "By 2035, the earth will become a supercomputer," which means that nearly everything on earth will be online or ready to be connected to the Internet [1]. Therefore, the term Internet of Things (IoT) has emerged, which can be a major player in the current digital world. The power of IoT technology not only shows the ability to connect billion of things via the Internet, but also this can be done automatically without human intervention. It did not stop here, as wireless technology developed, we approached what so-called the "Internet of Everything (IoE)"; which is a new concept that takes IoT to broader horizons, based on four elements: Things, People, Processes, and Data [2]. In Cisco's definition of IoE technology revolves around "bringing together people, processing, data and things to make network communications smarter and more valuable than ever, and transforming data into procedures that create new capabilities, richer experiences and an unprecedented economic opportunity for companies, individuals and countries" [2, 3].Moreover, IoE technologies are the fuel for the fourth industrial revolution, as they contribute effectively to linking trillions of objects and sensors, all of which generate real-time data. Thus, as it is said in our current digital age, "data is money", governments and companies can obtain the vast amount of information, provided they bring their strategies to process the big data and turn it into giant commercial and industrial businesses using different 
approaches $[4,5]$. Figure 1 shows the three critical expectations of IoE: scalability, intelligence, and diversity, which are motivate researchers to consider them in the Unmanned Aerial Vehicle (UAV) context. The Figure, also, emphasis the diversity of enabling technologies that can be considered to achieve these three expectations $[2,6]$.

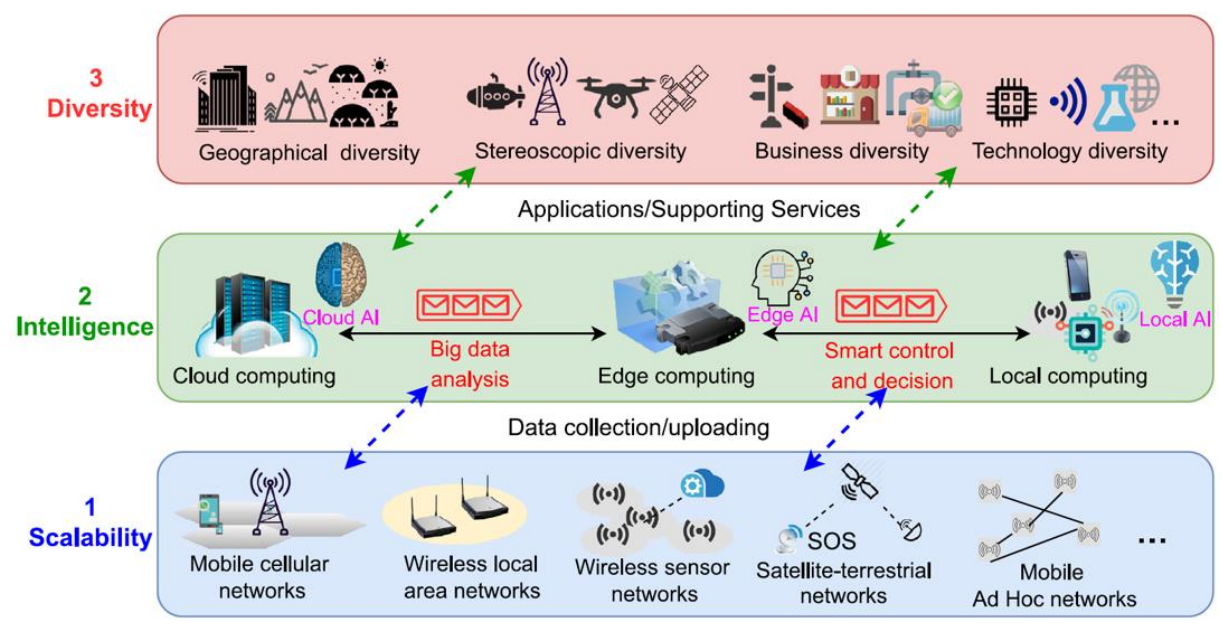

Figure 1. Three expectations of IoE [6].

Wireless communication services can be provided by either terrestrial and/or space-based systems includes UAV, satellites, or aerial balloon; which can be tethered balloon or flyballoon. Both terrestrial and satellite systems come with their own drawbacks. The former, has limitations of cellular systems dis-connectivity during natural disasters, coverage area gaps, deployment complexity especially in difficult terrain areas. Whereas the latter suffers from issues such as propagation delay, orbital maintenance, manufacturing and launch complexity, environmental damage resulting from gas emissions during launch, cost, and short satellite's lifetime due to gravitational pull and de-orbiting [7, 8]. However, UAV provides cost-effective infrastructure for long-term provision of broadband services to either fixed or mobile users. UAV considers as nearspace-satellites but without the distance, high cost or complexity downsides. Further, this environmentally friendly system can provide wireless services with a good level of connectivity and data capacity available for rapid deployment, network flexibility and reconfigurability with various dynamic coverage demands. This would not only enhance connectivity but also empower $\mathrm{IoE}$ and Fifth Generation (5G) technology with greater coverage footprint and line-of-sight adaptability [6]. UAV can, also, provide seamless services over heterogeneous networks to deliver a high QoS for global connectivity aimed at long-term or short-term large-scale events, which includes unplanned events (e.g. natural disasters). The rapid deployment of UAV wireless system has the advantages of being less vulnerable to infrastructure to be physical damaged during natural disasters. Thanks to fast start-up time infrastructure and direct connectivity to terrestrial users [7-9]. UAV term can include different types which might be High Altitude Platforms (HAPs), Low Altitude Platforms (LAPs), or even tethered aerial platforms. An aerial view of a UAV network architecture is shown in Figure 2, which includes space segment, and ground segment [7]. The former comprises a platform with its communication payloads; However, the latter consists of terrestrial users of both fixed and mobile users, besides a ground control centre along with wireless communication links within a covered footprint that can empower IoE applications. 


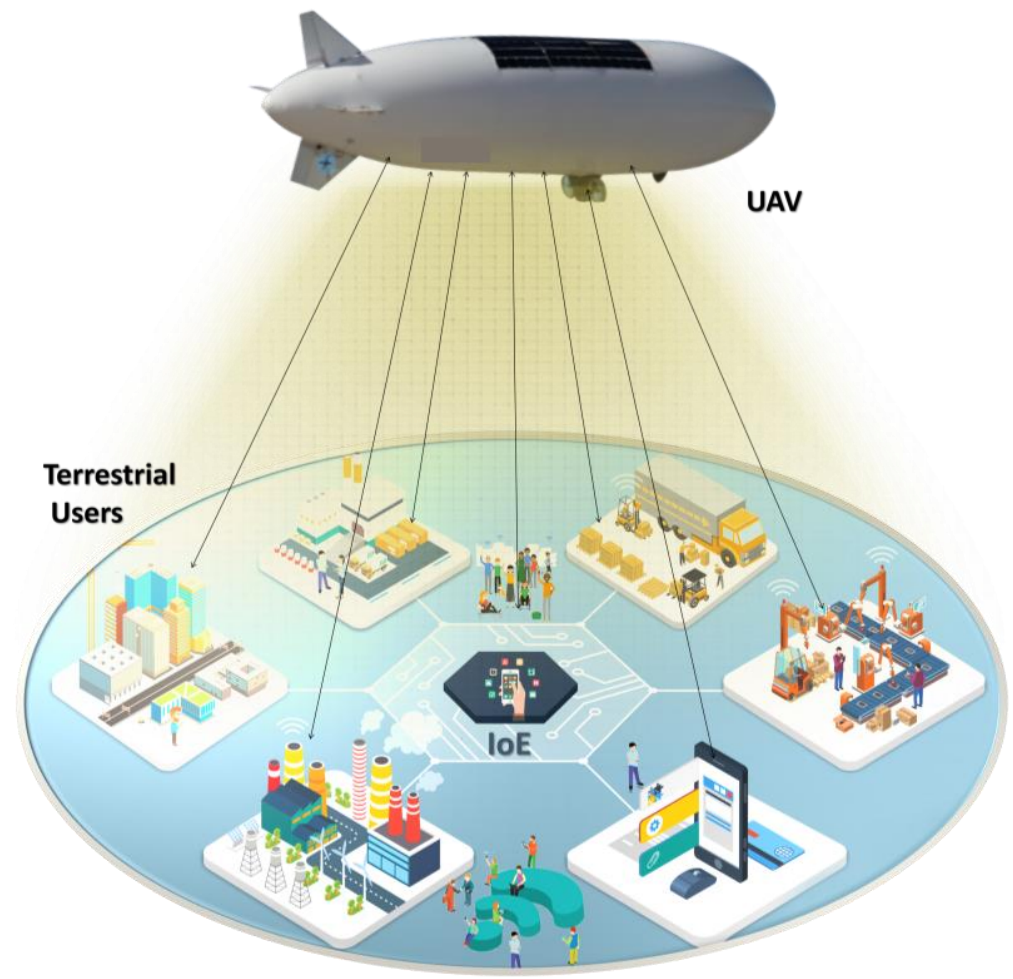

Figure 2. Aerial Vehicle-to-everything scenario.

Considering a study of aerial vehicle-to-everything wireless communication from is scarcely reported in the literature where it focuses on issues that allegedly can assess any wireless systems, let alone taking into consideration a combined propagation model of both indoor and outdoor that suit intense populated urban environment. Thus, the motivation of this paper is to provide an adaptive approach using a GA technique for combining indoor and outdoor propagation models to enhance aerial vehicle-to-everything wireless connectivity. The proposed adaptive approach uses a GA multi-objective function that yield optimum values of UAV altitude $\left(h_{\mathrm{t}}\right)$, elevation angles $(\theta)$, and type of building for indoor environment $(\mathrm{X})$. The GA optimization technique would meet the demand of a typical dense-populated urban environment, as well as empowering the IoE with greater coverage footprint, high QoS benchmark, and Lo'S adaptability. The rest of this paper organized as reviews related works on UAV technology, channel modelling, IoE, and machine learning (ML) techniques from different aspects in section II. Section III presents the proposed framework that uses GA approach to enhance aerial vehicle-to-everything wireless communications. Followed by section IV shows the simulation and implementation. Section V presents the results and discussion. Then, conclusion and future perspectives in section VI.

\section{RELATED STUDY}

The concept of using UAVs as aerial base stations to provide varies wireless services have already been addressed in the existing literature from different perspectives; Thus, we categorize them into subsections as follows: Propagation Model, Internet of Everything, and Optimization using Machine Learning. 


\subsection{Propagation Models}

Propagation models are widely deemed in wireless communications to calculate Path Loss (PL) and coverage via measuring the power density of electromagnetic waves as they propagate over space. These measurements are important for network planning as well as monitoring system performance. The propagation models have several factors that affect their performance (e.g. frequency, terrain, antenna heights of transmitter and receivers) [7, 10, 11]. Studying propagation path loss model types and characteristics that used for UAVs scenarios is vital, where radio signals propagate vis free space until reaching the complex ground ubiquitous environment with scattering and shadowing effects. Therefore, propagation models for UAVs technology reported in the literature are broadly based on either; First: Free Space Path Loss models (e.g. Air-To-Ground, and Two-Ray). Second: empirical propagation models (e.g. ITU indoor, and Okumura-Hata) [7-9]. Researchers in [12] addressed the effect of aerial platform altitude on wireless connectivity and coverage footprint, which is a vital element in any propagation model. The paper has considered a closed-form formula of free space propagation model which includes LoS and non-line-of-sight (NLoS) scenarios. Performance analysis of a propagation modelling based on elevation angle via HAP is introduced [13]. Results show variation in path loss values depend on geographical conditions (urban, suburban, or rural), as well as HAP's several altitudes. It recommends that more work should take place to reach an optimal design for different environment and elevation angle scenarios.

The work in [14] shade the light on propagation model as a function of elevation angle in different types of urban areas via HAPs mobile communications. The model includes two separated cases of Los and NLoS between the aerial platform and terrestrial terminals. Results show realistic implementation of such a shadowing model dependent on the element of elevation angle variation. Researchers in [15] highlighted the recent advancement and trends of UAVs for 5G communications at different altitudes. Such a technology can evolve dedicated sensors that can enable many applications for disaster relief (e.g. communication assistance, low altitude surveillance, and post-disaster rescue). The paper emphasis the cornerstone parameters of channel modelling of UAV namely; altitude, elevation angle, and environment probabilities. These parameters are vital for evaluating system performance. The research confirms that the harmony of IoT and UAVs remains unexploited field, which encourage researchers to conduct researches in it as a future work.

Free space propagation model is introduced in [16, 17, 18], where they proposed Air-To-Ground (ATG) path loss model between LAPs and terrestrial users. The model is highly dependent on the probability of receiving LoS and NLoS components with inclusion of ITU-R statistical parameters for different environments. The ATG model showed a clear tendency towards wide coverage footprint, reasonable shadowing effect. Results, also, emphasize the vital impact of elevation angle, beside LAP's altitude on the performance of ATG model, yet optimization is needed. The paper recommends that the Air-to-Indoor penetration model could be considered as future work for wider connectivity. Authors in [19] studied the behaviour of a Two Ray propagation model at high frequency $(28 \mathrm{GHz}$ and $60 \mathrm{GHz}$ ) for $5 \mathrm{G}$ wireless networks using UAV. The simulations have analyzed the results of Received Signal Strength (RSS) and root mean square delay spread (RMSDS) of multipath components (MPCs) for different environments: urban, suburban, rural, and oversea. Simulation results have shown that the Two Ray propagation model is promising to utilize high mmWave bands for aerial platform wireless communication for the sake of high capacity. Overcome issues like low UAV heights, atmospheric scatters are highlighted as further work recommended to improve RSS results.

Researches in [20-23] have focused on empirical propagation models (e.g. Hata, and Okumura) for various aerial platform wireless services. These models are heavily relying onpre-defined set of 
constants and constraints for different geographies. Regardless of accuracy of empirical propagation models results, these models exhibit some shortcomings as a result of their limited antenna heights and short transmission coverage. Further, empirical propagation models still immature for aerial platform applications in comparison to those used for terrestrial systems. Authors in $[24,25]$ have investigated both free-space propagation models (e.g. ATG), and empirical propagation models (e.g. Okumura) for the last mile connectivity using LAPs. The studies show both advantages and limitations to aspects that affect performance of any propagation models (e.g. elevation angle, altitude, operational frequency, antenna specifications, and power consumption). From the obtained predictions of various models, a machine learning approach is proposed to evolve a propagation model that can achieve better coverage probability, moderate power consumption, and more Los connectivity. A step further was taken in $[26,27]$ where they focused their attention on conceiving an intensive survey on large-scale and small-scale fading channel models including their restrictions, and future research directions for UAV wireless communication services. What even more, authors in $[28,29]$ have covered a propagation model for indoor environments using UAVs. However, neither small-scale fading channel model nor indoor propagation model has been considered collectively with either large-scale fading channel model or outdoor propagation model, respectively. Thus, it is indorsed that more elaboration is needed for hybrid models that contains both propagation types as part of the future work.

\subsection{Internet of Everything}

Nowadays in the fast-growing era of interactive and informative intelligent devices (smartphones, ships, smart sensors, autonomous vehicles, etc.), where these devices are seamlessly embedded in our daily lives and connecting the unconnected. The concept of Internet of X headed beyond smart devices to achieve replete technology that has enabled both synchronous and asynchronous communication without human interaction in various directions in this digital live (e.g. Home, Energy, Tourism, Industry, Transportation, Environment, Health care, Security, and Automotive)[2, $6,30]$. Hence, the IoE is a new concept that takes IoT to broader horizons, based on four elements: People, Processes, Data, and Devices. People: Connecting persons in more appropriate ways. Process: Providing accurate information to the right person and/or machine at the right time. Data: Converting raw data into meaningful and intelligence information to make truthful decisions. Devices: Physical devices are connected via the Internet, which called "IoT" [2-6].UAVs have promising potential to revolutionize the future of enabling IoE by working as wireless aerial relays to improve connectivity to ground networks, people, and devices. In [6], the article presents an intensive review on opportunities and challenges of UAV-enabled IoE along with enabling technologies for three expectations of IoE (i.e., scalability, intelligence, and diversity).

The authors of [31, 32] shade the light on IoT-enabled applications via UAVs technology (e.g. smart home/cities/ retail/grids/farming, connected health, wearables, connected vehicles, industrial internet) that can be enabled to get more connected societies. Researchers in [33] proposed architecture of UAV-enabled healthcare includes discussing open research issues and outline the future directions of Use-IoE. The UAV-based smart healthcare framework comprising three operations between UAVs and multiple Body Area Networks (BANs) connected to patients, which is data collection, data reporting, and data processing; then the servers process the patients' information to make health decisions includes real-time diagnosis and prescription. The performance results emphasis an acceptable data flow, throughput and delay. The article identified open research issues in UAV-based data gathering in smart healthcare systems (e.g. coverage, MAC layer, routing layer, localization, and handover procedures). Another field of UAV capability in empowering IoE is covered in [34], where the article highlights distributed aerial processing via UAV for IoT smart farming. The proposed work considered a Nash bargaining-based weighted intra-Edge processing offload scheme to mitigate the problem of heavy processing for various 
network topologies. The results show a positive performance regarding processing speed-ups, as well as scalability of deployment of UAV-enabled IoT.

Authors in [35, 36] have worked on communication architecture that satisfies the requirements of $5 \mathrm{G}$ connectivity of mobile users and IoT devices. Taking advantage of the UAVs network's flexibility of moving radio access (RA) nodes to zones where users struggle due to poor connectivity and/or low data rate. ML techniques based on a pattern recognition framework used in [35] for decision-making capabilities to maintain network connections, as well as process and distribute data. A Multiple-Input and Multiple-Output (MIMO) antenna for UAV to everything communication is, also, introduced to maximise capacity, improve coverage extension range, and reduce power consumption, which in turn meet the reliability of aerial platforms. Smart cities comprise smart things which can automatically and collaboratively enhance life quality and save people's lives. To achieve such tasks, reliable technologies such as UAVs, Artificial Intelligent (AI), and IoT are required to increase the smartness of smart cities via enhancing connectivity, and energy efficiency [5]. Further application of UAVs is presented in [25, 37], where UAVs act as an aerial ad hoc network for rapid restoration of communications links after disasters. The prediction results show that UAVs can offer robust communication links during emergency relief operations. Such a rapid deployment of UAVs to everything connections can be the fine line between life or death in disasters situations.

\subsection{Optimization using Machine Learning}

To enhance the performance of a model, an optimization concept using Air-To-Ground techniques should be considered. ML algorithms and techniques are used for data analytics to obtain valuable information from complex and large data that allow making a smart decision. The learning concept is based on learning from the internal patter of the data, where data can adjust their internal parameters accordingly. Further, Artificial Neural Networks (ANN), and GA are the most candidates that could be considered, as they are very complex and attractive techniques that have been used widely for various optimization work [39, 40]. ANN techniques have been widely applied to many different systems analyses and optimization issues. Moreover, ANN can be a wellsuited and high-quality tool for smart decision- making as a classification problem. Where ANN consists of more than one neuron that trained to predict optimum output based on an algorithm and some input elements. The three basic elements of a neuron are the synaptic weights, the summing junction, and the activation function. Once an ANN network created for a specific application, the process begins by arbitrarily choosing the elementary weights, where the training can be either supervised or unsupervised. The processing time is subject to the size of a neural network. Process of ANN stops where either threshold value or the number of iterations is reached. ANN can develop its organization (self-organization), generalize, and tolerate faults [21, 39].

On the other hand, GA is a search technique used in computing to find true or approximate solutions to optimization and search problems, taking advantage of MATLAB interactive environment to develop an algorithm that solves a variety of optimization problems. In this technique, after generating a prime population of solutions, at the first step, the fitness of all solutions gets described then reproducing the most successful solution, which is done by applying the stochastic operators, this is (Selection). Recombining separate solutions (Crossover) and randomly mutate solutions (Mutation). Process of GA stops where either a maximum number of generations have been generated, or a required fitness rate has been achieved for the population. GA can well-performed over a broader class of problems, and overs multiple solutions. Moreover, it is an easy method to understand and suitably transfer to existing simulations and models [40, 41]. As part of their continuous efforts, researchers strive to introduce various ML optimization techniques to enhance the performance of aerial platforms system and deliver seamless wireless communication services. Authors in [21, 24, 25, 37, 42, 43] have investigated the optimal 
propagation model using different types of ANN techniques (e.g. Self-Organizing Map (SOM), NNs Feedback Forward fitting tool, and NN Radial Basis Function (RBF)). Where the aim of using these ML optimization techniques were mainly to obtain higher LoS connectivity, wider coverage and decrease the power consumption. These papers could open a new direction of research and development in the area of propagation models optimization using ML approaches. Authors in [44] have considered a Self-Organizing Network (SON) to achieve the goals of self-configuration, selfoptimization, and self-healing of aerial base station using an AI method. The AI framework aims to adjust the optimal communication parameters according to user requirements and also to enhance the wireless connectivity, QoS, and aerial imaging that help for crowed management. The work in [45] shows optimization of UAVs trajectories, collision avoidance, and energy efficiency using deep reinforcement learning (RL) algorithms. Results confirm an improvement in link budget parameters of NN optimised model.

Researchers in [46] proposed a NN self-healing model to maximize the UAVs positioning likelihood for optimized throughput, coverage and maximum UE to UAV mapping in 5G networks. Results show that the performance of a neural model was an efficient approach based on the upper and lower values for the UAVs' positioning likelihood along with energy consumption efficiency. Authors in $[47,48]$, present a framework for the successive convex approximation (SCA) technique to maximize throughput in UAV-enabled mobile relaying with multiple source nodes. The SCA optimization is jointly optimised the transmit power, time slot scheduling, and UAV trajectory. Numerical results obtained show that the proposed framework was effective and can bring a significant performance gain compared to the current benchmarks. [49] addressed a multi-objective problem of UAVs deployment that includes several parameters: number of UAVs, locations, transmitting power, and caching. The paper uses reinforcement learning to guarantee the QoS requirements and provide high-quality communication services on one hand; On the other hand, maintaining the optimal trade-off between power consumption and backhaul saving.

\section{Proposed Model}

Considering communications between UAVs to everything scenario with a combined indoor and outdoor propagation model is scarcely reported in the literature, let alone considering an adaptive optimised tool to enhance its performance. This proposed model along with its optimization framework aims to provide an adaptive approach using a GA technique for a combined indoor and outdoor propagation models to enhance aerial vehicle-to-everything wireless connectivity. The proposed adaptive approach uses a GA multi-objective function that yield optimum values of UAV altitude $\left(h_{\mathrm{t}}\right)$, elevation angles $(\theta)$, and type of building for indoor environment $(\mathrm{X})$. The GA optimization technique would meet the demand of a typical dense-populated urban environment, as well as empowering the IoE with greater coverage footprint, high QoS benchmark, and LoS adaptability via UAV wireless system.

Figure 3 shows there are two stages in our proposed model. The First stage is responsible for generating predicted results of the combined channel model, which contains two selected models collectively together. Taking into consideration selected inputs: $h_{\mathrm{t}}, \theta$, and $\mathrm{X}$. Then, based on the obtained predicted PL, a set of vital performance indicators parameters are considered; namely: RSS, throughput (T), and coverage footprint (D). The Second stage is responsible for the optimization process, where GA uses predictions obtained from the combined propagation models in stage one, and then solve a multi-objective based on assigning a weight to each normalized objective function. This optimization requires a minimax technique with assigned weights for different input parameters in an urban environment. Such an optimization approach should yield optimum values of the inputs, where the desired optimization features are planned to minimize path loss, transmission power and power consumption on one hand; On the other hand, maximize RSS, $\mathrm{T}$, and $\mathrm{D}$. 


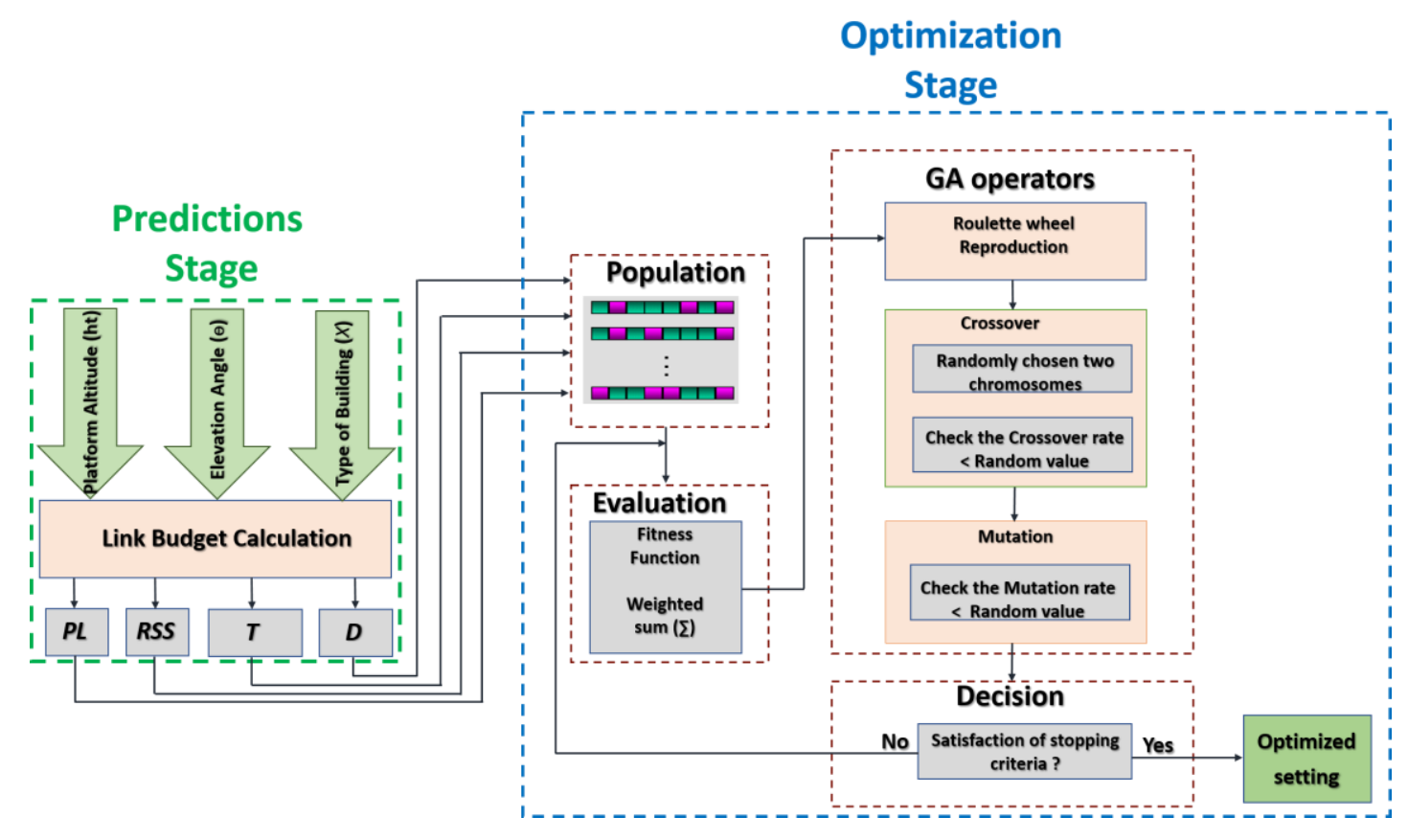

Figure 3. The Proposed Model.

\subsection{Combined Channel Model}

In the last few years, there were many competing propagation models have been considered for aerial platforms; yet whilst they exhibit numerous advantages, they also collectively raise many disadvantages. This proposed channel model aims to use two-channel models which are: Two-Rays propagation model as an outdoor model; and ITU indoor attenuation model as an indoor model. These two models collectively represent large-scale, and small-scale propagation models, respectively. Therefore, we target to cover this untapped research area of combining large-scale, and small-scale and similarly, attempt to increase IoE connectivity in a dense urban environment. Further, such a combined channel model exhibits advantages related to high altitude, wide coverage range, adaptation across outdoor and indoor urban environments. Figure4 illustrates the approximate channel model of the conceptual advantage of the proposed combined (indoor and outdoor) propagation model. Where a UAV can deliver connections to terrestrial users, which experiencing LoS and NLoS obstacles could cause diffracted, reflection, scattering. The proposed combined model takes into consideration various elevation angles along with altitudes between the $\mathrm{UAV}$ and mobile/fixed terrestrial users, which in turn leads to not only enhance connectivity but also support peer-to-peer (P2P) connections. 


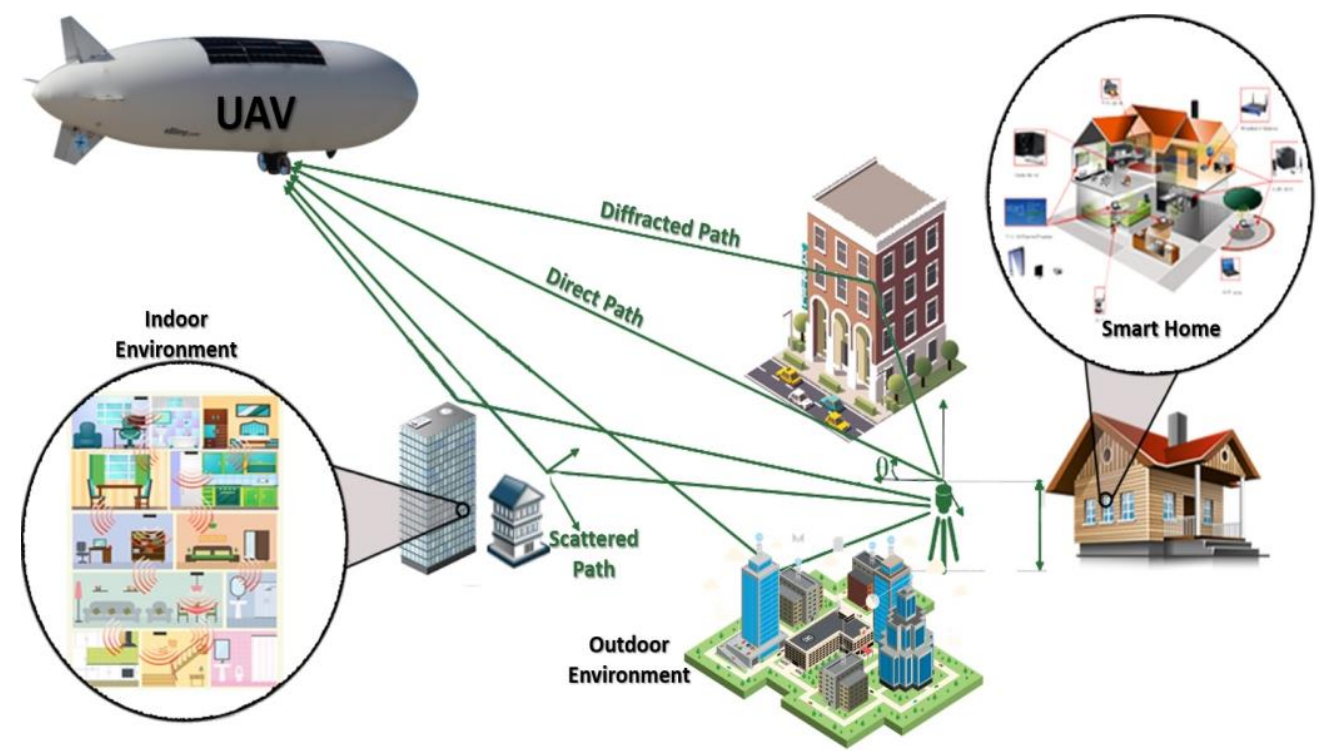

Figure 4. The proposed propagation scenario from a UAV to everything.

For the outdoor propagation model, we use the Two-Rays model, where path loss is critically dependent on the elevation angle [50]. The path loss of the Two-Rays propagation model is expressed as per equations (1) and (2):

$$
P L[\mathrm{~dB}]=40 \log (\mathrm{d})-\left[10 \log \left(G_{\mathrm{t}}\right)+10 \log \left(G_{\mathrm{r}}\right)+20 \log \left(h_{\mathrm{t}}\right)+20 \log \left(h_{\mathrm{r}}\right)\right]
$$

where the distance $d$ of the propagation model's range is computed based on elevation angle $\theta$ as follows:

$$
\mathrm{d}[\mathrm{km}]=2 E_{r}\left[\cos ^{-1}\left(\frac{E_{r}}{E_{r}+h_{\mathrm{t}}} * \cos (\theta)\right)-\theta\right]
$$

whereby given a UAV's altitude $h_{\mathrm{t}}, E_{r}$ is the Earth's radius at $6378 \mathrm{~km}$, and $\theta$ is the elevation angle from a user's location, $\operatorname{Gt}\left(h_{t}\right)$ : transmitter antenna height gain $(\mathrm{dB}), \operatorname{Gr}\left(h_{r}\right)$ : receiver antenna height gain $(\mathrm{dB}), h_{t}$ : transmitter antenna height $(\mathrm{m})$, and $\mathrm{h}_{\mathrm{r}}$ : receiver antenna height $(\mathrm{m})$ [50]. On the other hand, for indoor propagation model, we use the ITU model for indoor attenuation [50]. Where path loss is critically dependent on an average path loss along with its associated shadow fading statisticsindoor. Thus, the path loss of the ITU model for indoor attenuation is expressed as per equations (3):

$$
P L[\mathrm{~dB}]=20 \log _{10}(f)+N 10 \log _{10}(d)+L_{f}(n)-28(3)
$$

where $f$ is frequency $(\mathrm{GHz}), N$ is distance power loss coefficient, $d$ a separation distance $(\mathrm{m})$ between the transmitter indoor and receiver, $L_{f}$ is the floor penetration loss factor $(\mathrm{dB})$, and $n$ represents the number of floors between transmitter indoor and receiver. Typical parameters of $N, L_{f}$, and $n$ are given based on various measurement results, as Table 1 tabulates the values [50]. Those values are vital to predict indoor propagation of residential and commercial scenarios. 
International Journal of Computer Networks \& Communications (IJCNC) Vol.13, No.2, March 2021

TABLE 1: Values $\mathrm{N}$ and of ITU model for indoor attenuation.

\begin{tabular}{|c|c|c|c|}
\hline Parameter & Residential area & Office area & Commercial area \\
\hline N & N/A & 28 & 22 \\
\hline$L_{f}$ & $4 \mathrm{n}$ & $15+4(\mathrm{n}-1)$ & $6+3(\mathrm{n}-1)$ \\
\hline
\end{tabular}

Besides $P L$ calculation of the proposed model, RSS, $T$, and Dhave been included, as these parameters are considered as vital performance indicators for network planning, and QoS results. These link budget parameters can be predicted as per equations (4) to (6):

$$
\begin{aligned}
\mathrm{RSS}= & \mathrm{P}_{\mathrm{t}}+\mathrm{G}\left(\mathrm{h}_{\mathrm{t}}\right)+\mathrm{G}\left(\mathrm{h}_{\mathrm{r}}\right)-\mathrm{P}_{\mathrm{L}}-\mathrm{L} \\
& \text { SNIR }=\frac{\text { RSS }}{\mathrm{N}+\mathrm{I}} \\
& \mathrm{T}=\mathrm{B} \times \log (1+\mathrm{SNIR})
\end{aligned}
$$

where transmitter power $\left(P_{t}\right),(L)$ connector and cable loss, $N$ : Noise Figure $(\mathrm{dB})$ plus $I$ : Interference (dB), $D(\mathrm{~b} / \mathrm{s})$, bandwidth (B) in (MHz), SINR (dB) [24, 25].

\subsection{Optimization using Machine Learning}

Although, many optimization techniques are under consideration by number of researchers, and progressing rapidly, however, still miles to go for finding the best solution that can fit all problems. Where many researchers confirm that is no one size algorithm and/or optimization method can fit all problems. Moreover, these optimization techniques do not have all the advantages together, where some disadvantages reduce their efficiency. Thus, after many tests have been done, we end up with a well-suited, reliable and well-performing model that fulfil our requirements using the proposed GA approach in the shortest time. The technique of solving a multi-objective optimization considered here is called the "Weighted sum approach". This classical approach is based on assigning a weight to each normalized objective function, hence converted the problem to a single objective problem with a scalar objective function. Then, randomly generating an initial set of solutions and evolving these solutions over time and convert them into a single solution to eventually generate an optimal solution. This requires a minimax technique with assigned weights for different parameters at various UAV altitudes and elevation angles in an urban environment [40, 41]. As Figure 3 shows that it is commonly known that after generating a prime population of solutions, at the first step, the fitness of all solutions gets described then reproducing the most successful solution, which is done by applying the stochastic operators, this is (Selection). Recombining separate solutions (Crossover) and randomly mutate solutions (Mutation). Process of GA stops where either a maximum number of generations have been generated, or a required fitness rate has been achieved for the population [37, 40, 51- 53]. The fitness function is considered to alter the objective function value into a measure of relative fitness, hence it can be expressed as per equations (7) to (10):

$$
F(x)=g(f(x))
$$

where $f$ is the objective function, $g$ transforms the value of the objective function to a non-negative number and $F$ is the resulting relative fitness. A fitness function, $F(x)_{i}$, measures individual fitness as the individual's raw performance, $f(x)_{i}$, relative to the whole population:

$$
\mathrm{F}(\mathrm{x})_{\mathrm{i}}=\frac{\text { fobj }(\mathrm{x})_{\mathrm{i}}}{\sum_{\mathrm{i}=1}^{\mathrm{n}_{\text {nind }}} \text { fobj }(\mathrm{x})_{\mathrm{i}}}
$$


where $n_{\text {ind }}$ is the population size and $x_{i}$ is the phenotypic value of individual $i$. Multi-objective optimization is rather a realistic formulation to solve many complex engineering problems. The problem is converted to a single objective problem with a scalar objective function as follows:

$$
\begin{array}{r}
\text { fobj }=\sum_{i=1}^{\mathrm{m}=1} \mathrm{~W}_{\mathrm{i}} \text { fobj, } \mathrm{i}\left(\mathrm{a}_{1}, \mathrm{a}_{2}, \ldots . \mathrm{a}_{\mathrm{r}}\right) \\
1 \geq \mathrm{W}_{\mathrm{i}} \geq 0 \text { for } \mathrm{i}=1,2, \ldots, \mathrm{m} \\
\mathrm{W}_{1}+\mathrm{W}_{2}+\cdots+\mathrm{W}_{\mathrm{m}}=1
\end{array}
$$

where, $a_{1}, a_{2}, \ldots . a_{r}$ is a list that represents the input parameters of objective functions and the set $W_{1}, W_{2}, \ldots, W_{m}$ satisfies the weight for each parameter (objective) that floats between an upper and a lower bound. Further, smart decision-making should rank all objectives according to the target of the application by designing the weight values to solve the problem of having more than one optimal. The GA solution space is generally restricted by a series of constraints for each parameter floats between an upper and lower bound. Therefore, in our case the formula can be written as follows:

$$
\text { fobj }=W_{1} * f o b j_{\min }(P L)+W_{2} * f o b j_{\max }(R S S)+W_{3} * f o b j_{\max }(T)+W_{5} * f o b j_{\max }(D)
$$

To meet the optimal solution, the GA should observe the minimax of the following parameters along with their assigned weights as: minimizing $P L$ and maximizing $R S S, T$, and $D$ at varies UAV's altitudes and elevation angles. The advantage of smart decision-making by optimizing would help to provide proper wireless services to a given environment.

\section{SIMULATION AND IMPLEMENTATION}

The proposed model has been simulated into two main stages using MATLAB. Stage one is responsible for generating predicted results of the combined channel model, which contains two selected models collectively together. Taking into consideration selected inputs: $h_{\mathrm{t}}, \theta$, and X. From this, predicted PL can be obtained. Subsequently, RSS, T, and D of the combined model can be predicted. Stage two is responsible for the optimization (adaptation) process, where it can be fed by initial prediction results of the combined propagation model. Then, solve the GA multi-objective based on assigning a weight to each normalized objective function. This optimization requires a minimax technique with assigned weights for different input parameters in an urban environment. Such an optimization approach should yield optimum values of the inputs, where the desired optimization features are planned to minimize PL, transmission power and power consumption in one hand; On the other hand, maximize the RSS, T, and D. These parameters are vital performance indicators for any wireless communication system. Following simulation parameters of a $5 \mathrm{G}$ MIMO antenna specification that obtained ITU Radiocommunication Study Groups for $4 \mathrm{GHz}$ frequency band [54]. Bandwidth [20 MHz], modulation type [256 QAM], Noise Figure [7 dBm], $h_{t}$ [0.2-5km], , $\mathrm{P}_{\mathrm{t}}[44 \mathrm{dBm}], \mathrm{G}\left(\mathrm{h}_{\mathrm{t}}\right)$ with diversity gain [20 dBi], transmitter $\mathrm{h}_{\mathrm{t}}$ sensitivity [-88.9dBm], Interference margin loss $[5.5 \mathrm{~dB}]$, losses $[0.8 \mathrm{~dB}], \mathrm{G}\left(\mathrm{h}_{\mathrm{r}}\right)$ with diversity gain [5 dBi], receiver $\mathrm{h}_{\mathrm{r}}$ sensitivity $[-88 \mathrm{dBm}]$, and $\mathrm{h}_{r}[1.5 \mathrm{~m}]$.

Broadly, there are standard indicators obtained from GA parameters; namely: population size, number of iterations, mutation rate, and crossover rate. Default values of the GA parameters are drawn from the literature and tested several times here can be summarized as follows: A population size is 300 , number of iterations is 2000 , mutation rate is 0.01 , and crossover rate is 0.66 . To note, too small population size lead to low improvement per iteration in the fitness function, however, too high means fitness function increases faster and does not improve. Thus, as rule of thumb is that 100 is a good value when number of variables $<=5$. The default value of mutation rate $\left(m_{r}\right)$ must be carefully set, where too low $m_{r}$ the algorithm will not cover the search space, yet too high 
International Journal of Computer Networks \& Communications (IJCNC) Vol.13, No.2, March 2021

respectable solutions will be troubled and transformed to worse solutions. Therefore, $m_{r}$ can be calculated as $\max \left(0.01, \frac{1}{N}\right)$, where $N$ is population size. The default value of crossover rate floats between floats between an upper (0.9) and a lower bound (0.5), hence the average is $(0.70)$. Figure5 shows the simulation set up and Graphical user interfaces (GUIs) using MATLAB for the proposed combined propagation model along with its parameters and output results. Figure 6 displays the genetic algorithm optimization framework using GUIs MATLAB tool.

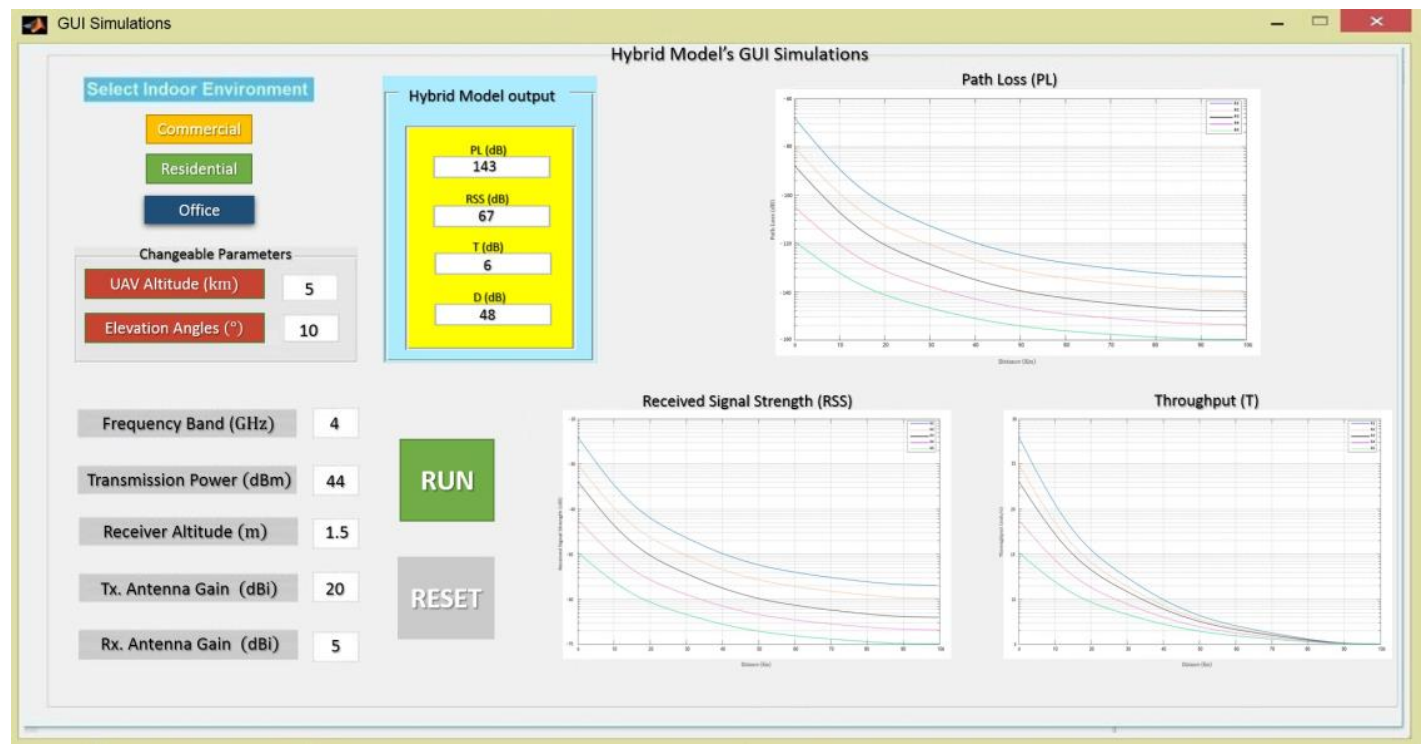

Figure 5. MATLAB GUI of the Proposed Combined Propagation Model.

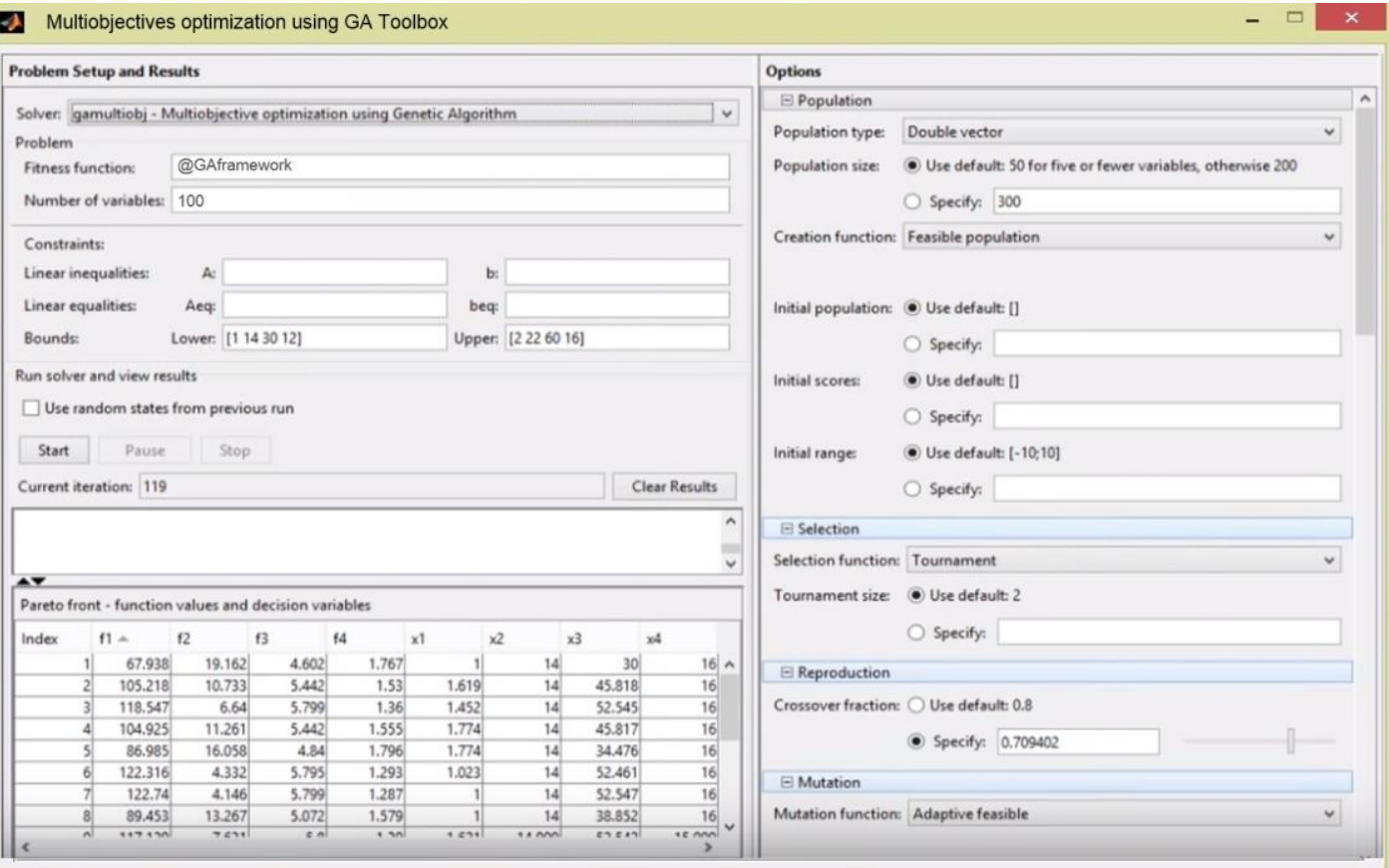

Figure 6. MATLAB toolbox for GA optimization solver. 


\section{RESUlTS AND DISCUSSION}

In this section, the results of the proposed model for the combined propagation model along with its GA optimization framework are presented and discussed. The results were analyzed using MATLAB, where several simulation runs have been performed for each stage of the proposed model, as following Figure7 to Figure13 show. The proposed framework model reveals several factors that are significant when considering UAV to everything connectivity. Firstly, input factors are all the antenna specification parameters mentioned in the prior section. All these input parameters have fixed values excluding: $h_{\mathrm{t}}, \theta$, and $\mathrm{X}$, are vary. Secondly, output parameters are PL, RSS, T, and D, where these parameters can help for monitoring network planning, and act as system performance indicators.

Figures 10-12 display the predictions of PL, RSS, and T, respectively, at various $h_{\mathrm{t}}$ of $0.5 \mathrm{~km}, 1 \mathrm{~km}$, $2 \mathrm{~km}, 10 \mathrm{~km}$, and $20 \mathrm{~km}$, respectively for $\theta=10^{\circ}$. These predicted results are selected ones out of hundreds of results driven from the simulations based on the following variations:

- $\theta$ ranges between $1^{\circ}$ to $90^{\circ}$

- $\mathrm{h}_{\mathrm{t}}$ ranges between $0.2 \mathrm{~km}$ to $5 \mathrm{~km}$

- Indoor scenario factors of $\mathrm{N}, \mathrm{L}_{\mathrm{f}}$, and $\mathrm{n}$ for residential area, office area, and commercial area

The output parameters of the combined propagation model are obtained by calculation the link budget parameters of the Two-Rays propagation model that collectively added to ITU indoor Attenuation model and deployed at various $\theta, h_{t}, N, L_{f}$, and $n$. These parameters are vital for evaluating network planning and assessing the performance of the proposed combined propagation model. Clearly in Figure7, the PL shows increases as distances increase, because the power density of an electromagnetic wave reduces as it propagates over space. The total path loss ranges between $-86 \mathrm{~dB}$ to $-150 \mathrm{~dB}$ across different scenarios.

Figure8 displays RSS which has a negative relation with PL, which means as PL value increases the RSS decreases. The RSS values vary between $-25 \mathrm{~dB}$ and $-70.5 \mathrm{~dB}$ which is less than $-85 \mathrm{~dB}$ threshold based on the link budget. An Increase in UAV altitudes and/or decrease elevation angles leads to an increase in distance which in turn leads to reducing throughput from $27 \mathrm{mb} / \mathrm{s}$ to around 8 $\mathrm{mb} / \mathrm{s}$ across different scenarios, as Figure9 shows. In contrast, an increase in UAV altitudes and/or decrease elevation angles results in increasing in distance, which varies between $3 \mathrm{~km}$ to $100 \mathrm{~km}$. It is, also, observed that increase in transmission power leads to increase path loss, as well as coverage footprint.

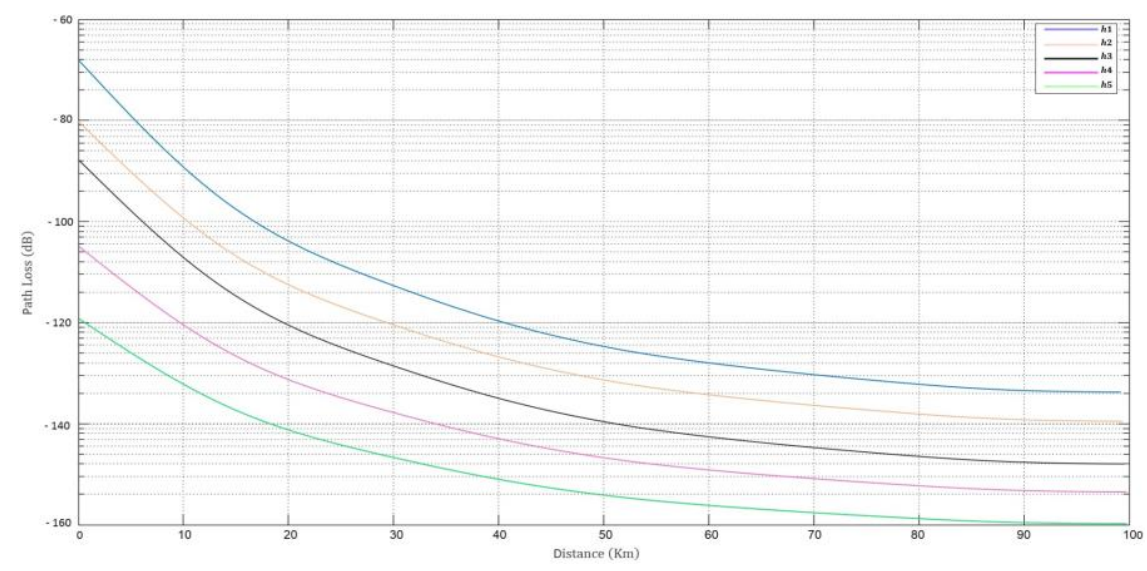

Figure 7. PL predictions at different UAV altitude. 


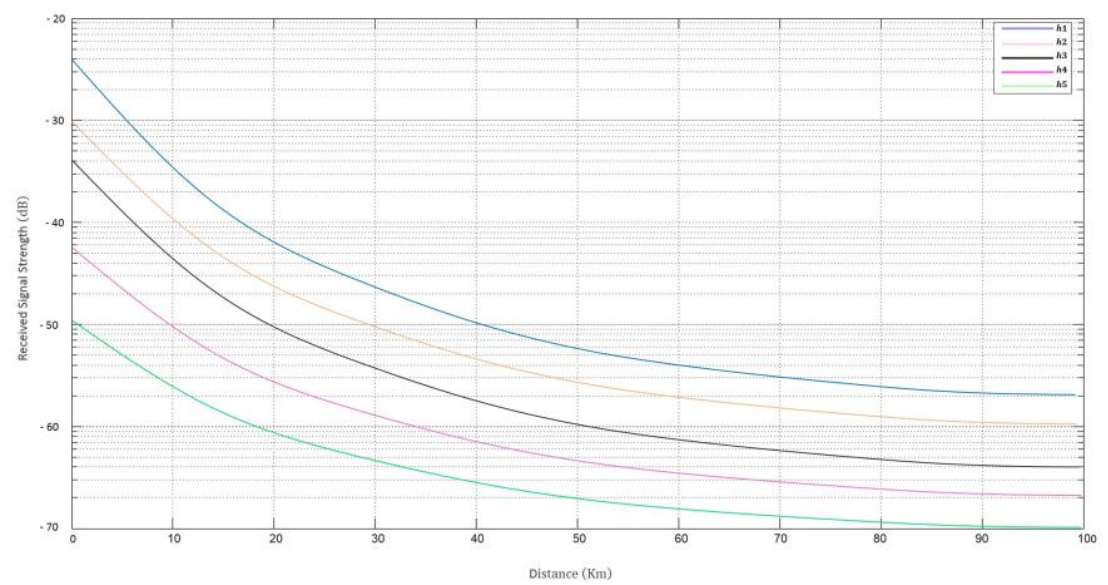

Figure 8. RSS predictions at different UAV altitude.

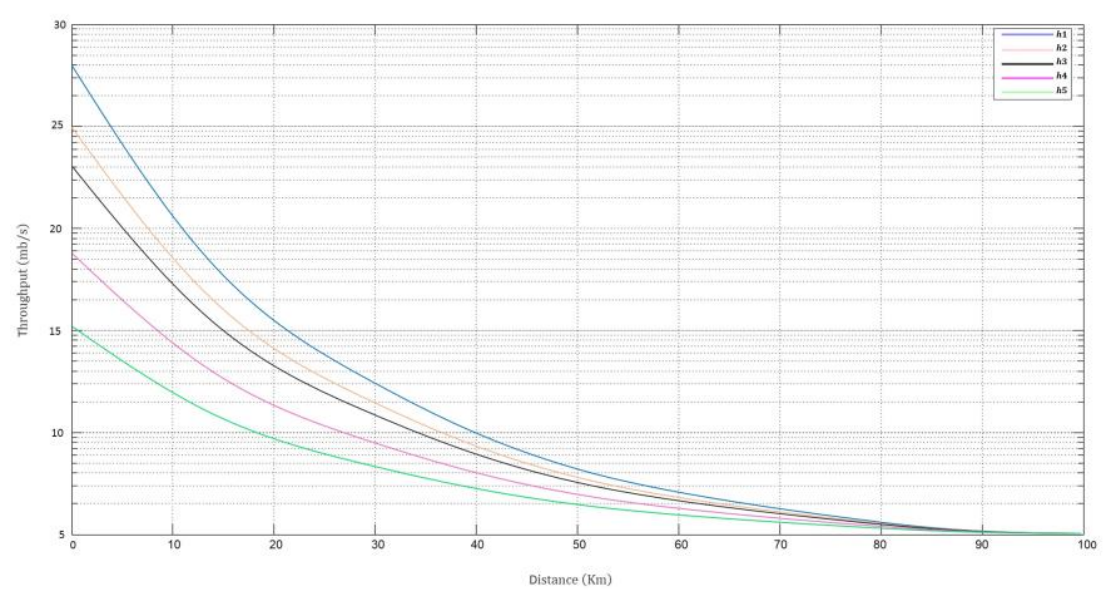

Figure 9. T predictions at different UAV altitude.

Figures 10-13 display the results of the GA optimization framework of the proposed model using MATLAB toolbox. The GA is based on a customized to solve the travelling salesman problem, which uses the GA minimax technique where the function value is smaller or larger at any other point in the search space. The travelling salesman problem is applied here where: each Gene stands for a possible optimal solution (represented as ( $\mathrm{x}, \mathrm{y}$ ) coordinates); Chromosome represents a single route between two solutions; Population stands for a collection of possible routes; Parents characterizes two routes that are combined to create a new route; Mutation: is the way to present variation in the population by randomly swapping optimal solutions in a route; Elitism stands for a way to transmit the best individuals into the next generation; Mating pool is responsible for collecting parents that are used to create our next population, then generating the next generation of routes. This article aims to use the GA optimization for data analytics to obtain valuable information from complex and large data that allow making a smart decision. The smart decision-making that aimed at is based on selecting the optimal solution of $\theta$, and $h_{\mathrm{t}}$ out of hundreds of solutions that inserted into the GA optimization model. To meet the optimal solution, the GA should observe the minimax technique of the following parameters as: minimizing PL, and maximizing RSS, T, and D at varies UAV's altitudes and elevation angles. Making smart decisions by the proposed optimization framework would help to provide proper wireless services to any given environment automatically without human intervention. 
Figure10 shows the progress distribution of all possible solutions using GA which apply global search method along with assigning the priority of data. While Figure11 displays the result of the optimization where optimal solutions are classified into different groups at iterations of 1264 . The classification refers to the initial possible solutions before the Pareto front takes place using socalled "Pareto-optimal solutions", where it relays on the best compromise solution. The Pareto front is deemed to be a wise strategy for multi-objective (multi-criteria) decision-making methods. Therefore, results in Figure11 obtained after searching all possible solutions than finding the closest one that achieve the fitness function using Pareto front that exists within the optimization MATLAB toolbox. The iteration number means the threshold of the fitness function achieves before reach the maximum iteration value.

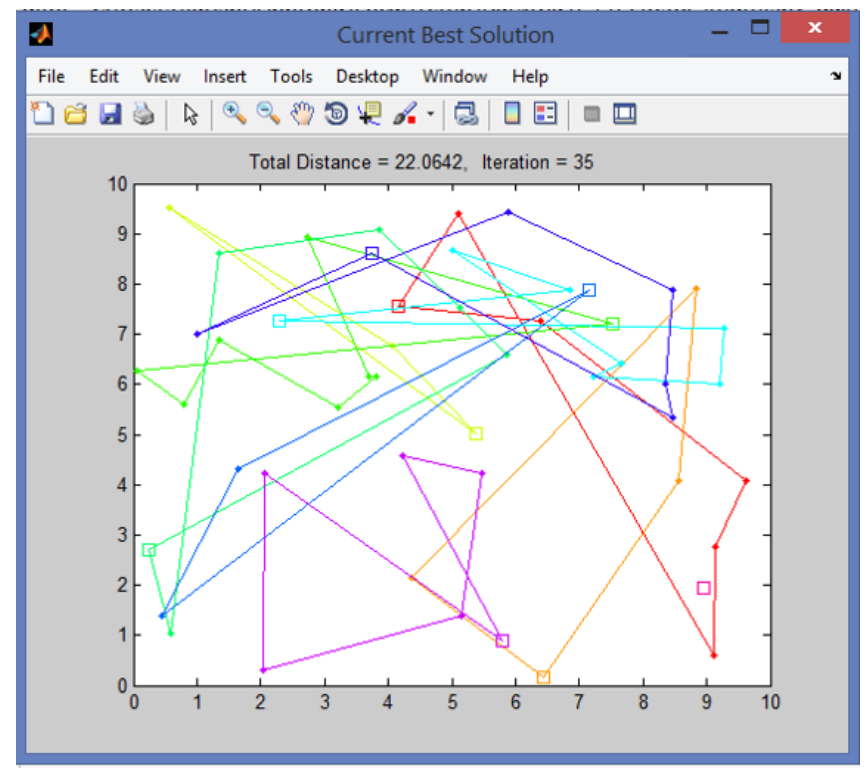

Figure 10. The progress of all possible solutions using GA.

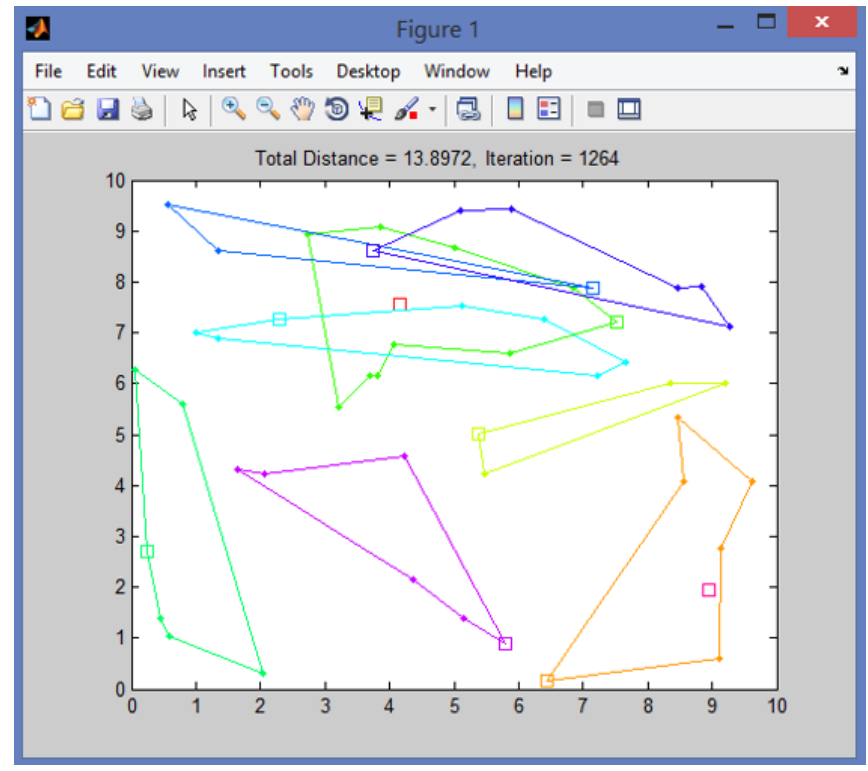

Figure 11. The classification of optimal solutions using GA. 


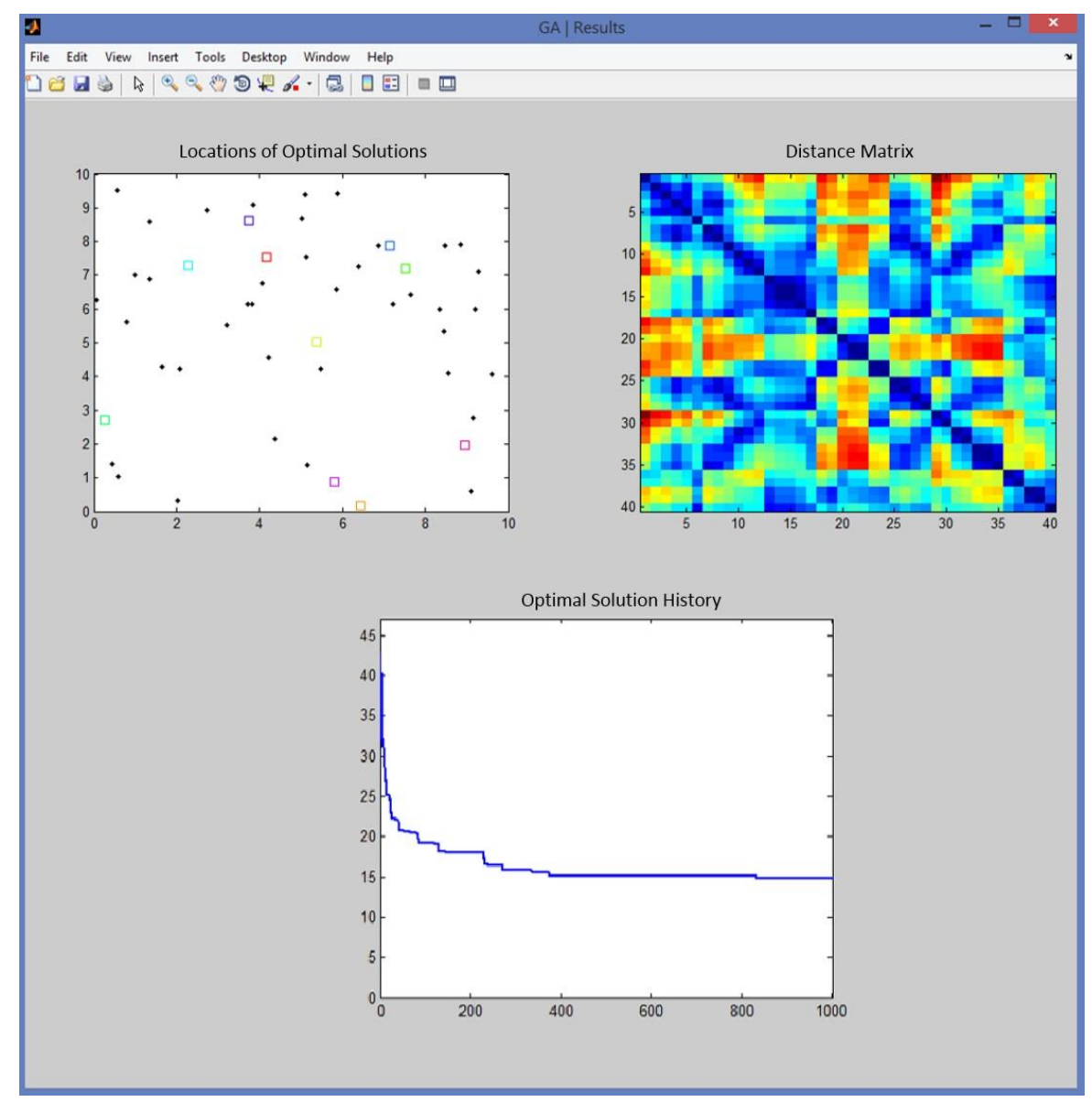

Figure 12. The optimal solutions of the proposed model using GA.

Results in Figure 12 confirm that each optimal solution has been visited at least once to be compared with the rest of the solutions based on the distribution of the locations of optimal solutions, as well as distance matrix. Also, the total distance in the matrix between optimal solutions has been calculated accordingly to check the connectivity between each pair of solutions, which shows reasonable connections in this Figure. The optimization execution time was 2671 seconds, where the optimal solutions obtained to make effective decisions. Therefore, the optimal solution is found when the fitness value is minimized. The graphical representation of Figure13 reflects how the proposed algorithm counts the feasible solutions, as well as converges to the optimal solution. The optimized parameters show an improvement with an average of $-5 \mathrm{dBm}$ in RSS, which in turns leads to improved coverage with $2.5 \mathrm{~km}$, and T with $3.4 \mathrm{mb} / \mathrm{s}$. Based on output results that is shown in Figures 10-13 are that the proposed GA optimization framework shows a well-suited and high-quality tool that is efficient for smart decision-making problems in a reasonable time manner. The smart decision-making that aimed at is based on selecting the optimal solution of $h_{t}$, and $\theta$ out of hundreds of solutions that inserted into the GA optimization model. Making smart decisions by the proposed optimization framework help in providing proper wireless services to any given environment automatically without human intervention. This has been achieved with a greater coverage footprint, less power consumption, high QoS benchmark and Los adaptability. 


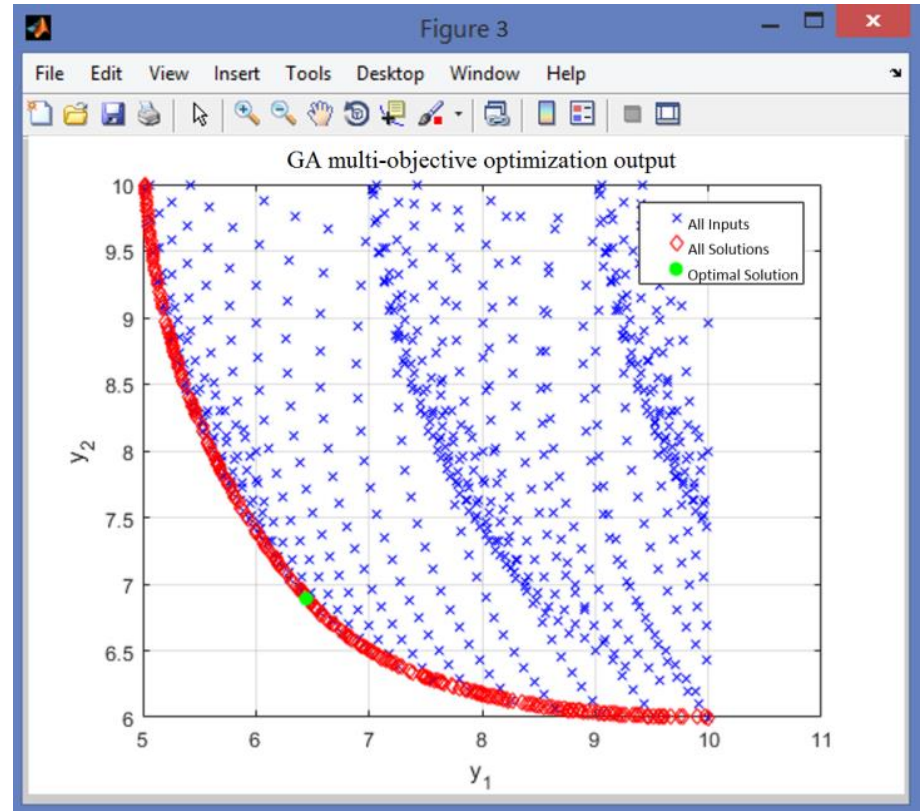

Figure 13.Validation of the GA multi-objective optimization output.

\section{CONClusion ANd Future Work}

The UAV technology is deemed to be an ambitious and promising wireless communication system due to its reliability and ease of deployment. The advantage of UAV includes, also, the ability to bridge the digital gaps and help in paving the way for IoE and 5G technologies, where billions of devices require wireless connectivity. Considering an optimized indoor-outdoor propagation model that include fine-tuning environmental considerations with the aid of GA has been the major motivation of this paper. The paper highlights the combined propagation model includes the TwoRay propagation model that collectively added with ITU indoor attenuation model into be deployed at various UAV altitudes and elevation angles. The combined propagation path loss along with other link budget parameters is entered to the GA optimization framework, which is aimed to enhance UAV to everything wireless connectivity by tuning the input parameters.

The proposed model along with GA optimization is analyzed over mobile 5G networks using MATLAB simulation toolboxes. The results of the simulated output can be concluded based on two main findings; First: the combined propagation has included various scenarios, which is a vital aspect to bridge the coverage gap / dead zones issue in any urban environment. Second: the GA optimization framework shows that it is a well-suited and high-quality tool for smart decisionmaking in a reasonable time manner. The output results emphasis that GA can find the optimum parameters set up to provide seamless connections via UAV, with greater coverage footprint, less power consumption, high QoS benchmark and LoS adaptability. The optimized parameters show, also, an improvement with an average of $-5 \mathrm{dBm}$ in RSS, which in turns leads to improved coverage with $2.5 \mathrm{~km}$, and T with $3.4 \mathrm{mb} / \mathrm{s}$. This work can be extended to develop an intelligent switch-unit in a real-time fashion that could select the best link budget parameters of UAV depends on a given urban, suburban, and rural environments and their ITU characteristics. Also, generating predictions in a 3D urban scenario is gaining momentum, as future work to provide more broaden results. 


\section{CONFLICTS OF INTEREST}

The author declares no conflict of interest.

\section{ACKNOWLEDGMENTS}

The author is grateful to the Deanship of Scientific Research at Taif University, Kingdom of Saudi Arabia for its approval and grants to this project (grants No. 1-440-6175).

\section{REFERENCES}

[1] "Technology QuarterlyThe Economist", The Economist, 2020. [Online]. Available: https://www.economist.com/taxonomy/term/90/0?page=83. [Accessed: 12- Mar- 2020].

[2] S. Mung, C. Cheung, K. Wu and J. Yuen, "Wideband Rectangular Foldable and Non-foldable Antenna for Internet of Things Applications", International Journal of Antennas and Propagation, vol. 2019, pp. 1-5, 2019.

[3] G. Marques, R. Pitarma, N. M. Garcia and N. Pombo, "Internet of Things Architectures, Technologies, Applications, Challenges, and Future Directions for Enhanced Living Environments and Healthcare Systems: A Review", Electronics, vol. 8, no. 10, p. 1081, 2019.

[4] M. Miraz, M. Ali, P. Excell and R. Picking, "Internet of Nano-Things, Things and Everything: Future Growth Trends", Future Internet, vol. 10, no. 8, p. 68, 2018.

[5] S. Alsamhi, O. Ma, M. Ansari and F. Almalki, "Survey on Collaborative Smart Drones and Internet of Things for Improving Smartness of Smart Cities", IEEE Access, vol. 7, pp. 128125-128152, 2019.

[6] Y. Liu, H. Dai, Q. Wang, M. Shukla and M. Imran, "Unmanned aerial vehicle for internet of everything: Opportunities and challenges", Computer Communications, vol. 155, pp. 66-83, 2020.

[7] F. A. Almalki, "Comparative and QoS Performance Analysis of Terrestrial-aerial Platforms-satellites Systems for Temporary Events", International Journal of Computer Networks \& Communications, vol. 11, no. 6, pp. 111-133, 2019.

[8] F. A. Almalki, M. C. Angelides, "Considering near space platforms to close the coverage gap in wireless communications; the case of the Kingdom of Saudi Arabia," FTC 2016 San Francisco - Future Technologies Conference, 2016, pp.224-230.

[9] M. Mozaffari, W. Saad, M. Bennis, Y. Nam and M. Debbah, "A Tutorial on UAVs for Wireless Networks: Applications, Challenges, and Open Problems", IEEE Communications Surveys \& Tutorials, pp. 1-1, 2019.

[10] A. Alotaibi and M. Angelides, "A serious gaming approach to managing interference in ad hoc femtocell wireless networks", Computer Communications, vol. 134, pp. 163-184, 2019.

[11] F. A. Almalki and B. O. Soufiene, "EPPDA: An Efficient and Privacy-Preserving Data Aggregation Scheme with Authentication and Authorization for IoT-Based Healthcare Applications," Wireless Communications and Mobile Computing, vol. 2021, pp. 1-18, Mar. 2021.

[12] A. Al-Hourani, S. Kandeepan and S. Lardner, "Optimal LAP Altitude for Maximum Coverage", IEEE Wireless Communications Letters, vol. 3, no. 6, pp. 569-572, 2014.

[13] Z. Hasirci and I. H. Cavdar, "Propagation modeling dependent on frequency and distance for mobile communications via high altitude platforms (HAPs)," 35th International Conference on Telecommunications and Signal Processing (TSP), Prague, 2012, pp. 287-291.

[14] J. Holis and P. Pechac, "Elevation Dependent Shadowing Model for Mobile Communications via High Altitude Platforms in Built-Up Areas", IEEE Transactions on Antennas and Propagation, vol. 56, no. 4, pp. 1078-1084, 2008.

[15] B. Li, Z. Fei and Y. Zhang, "UAV Communications for 5G and Beyond: Recent Advances and Future Trends", IEEE Internet of Things Journal, vol. 6, no. 2, pp. 2241-2263, 2019.

[16] A. Al-Hourani, S. Kandeepan, and A. Jamalipour, 'Modeling air-to-ground path loss for low altitude platforms in urban environments', IEEE Global Communications Conference, Austin, USA, Dec. 2014, pp. 2898 - 2904.

[17] M. Mozaffari, W. Saad, M. Bennis, and M. Debbah, "Drone small cells in the clouds: Design, deployment and performance analysis," IEEE Global Communications Conference, San Diego, USA, Dec. 2015, pp.16.

[18] G. E. Athanasiadou and G. V. Tsoulos, "Path Loss characteristics for UAV-to-Ground Wireless Channels," 13th European Conference on Antennas and Propagation, Krakow, Poland, 2019, pp. 1-4.

[19] W. Khawaja, O. Ozdemir and I. Guvenc, "UAV Air-to-Ground Channel Characterization for mmWave Systems," 2017 IEEE 86th Vehicular Technology Conference, Toronto, ON, 2017, pp. 1-5. 
[20] S. A. Khaleefa, S. H. Alsamhi, and N. S. Rajput, 'Tethered balloon technology for telecommunication, coverage and path loss', 2014 IEEE Students' Conference on Electrical, Electronics and Computer Science, Bhopal, India, Mar. 2014, pp.1-4.

[21] F. A. Almalki, M. C. Angelides, "Empirical Evolution of a Propagation Model for Low Altitude Platforms," IEEEComputing Conference 2017, 2017, pp.1297-1304.

[22] S. H. Alsamhi, M. S. Ansari, O. Ma, F. Almalki, and S. K. Gupta, "Tethered Balloon Technology in Design Solutions for Rescue and Relief Team Emergency Communication Services," Disaster Medicine and Public Health Preparedness, pp. 1-8, May 2018.

[23] S. Alsamhi, F. A. Almalki, S. Gapta, M. Ansari O. Ma, M. Angelides, "Tethered Balloon Technology for Emergency Communication and Disaster Relief Deployment", Springer Telecommunication Systems, 2019.

[24] F. A. Almalki, M. C. Angelides, "Evolution of an Optimal Propagation Model for the Last Mile with Low Altitude Platforms using Machine Learning", Elsevier Computer Communications Journal, vol. 142143, pp. 9-33, May 2019.

[25] F. Almalki and M. Angelides, "Deployment of an aerial platform system for rapid restoration of communications links after a disaster: a machine learning approach", Computing, vol. 102, pp. 829-864, Nov. 2019.

[26] W. Khawaja, I. Guvenc, D. Matolak, U. Fiebig and N. Schneckenburger, "A Survey of Air-to-Ground Propagation Channel Modeling for Unmanned Aerial Vehicles", IEEE Communications Surveys \& Tutorials, vol. 21, no. 3, pp. 2361-2391, 2019.

[27] S. A. Busari, K. M. Saidul Huq, S. Mumtaz and J. Rodriguez, "Impact of 3D Channel Modeling for UltraHigh Speed Beyond-5G Networks," 2018 IEEE Globecom Workshops (GC Wkshps), Abu Dhabi, United Arab Emirates, 2018, pp. 1-6.

[28] A. Aldhaibani, T. Rahman and A. Alwarafy, "Radio-propagation measurements and modeling in indoor stairwells at millimeter-wave bands", Physical Communication, vol. 38, p. 100955, 2020.

[29] Q. Zhang, G. Niu and M. Pun, "Large-Area Super-Resolution 3D Digital Maps for Indoor and Outdoor Wireless Channel Modeling," 2018 IEEE 87th Vehicular Technology Conference (VTC Spring), Porto, 2018, pp. 1-6.

[30] F. Almalki, "Utilizing Drone for Food Quality and Safety Detection using Wireless Sensors," 3rd IEEE International Conference on Information Communication and Signal Processing (ICICSP 2020), 2020, pp.238-243.

[31] F. Al-Turjman, M. Abujubbeh, A. Malekloo and L. Mostarda, "UAVs assessment in software-defined IoT networks: An overview", Computer Communications, vol. 150, pp. 519-536, 2020.

[32] N. Nomikos et al., "A UAV-based moving 5G RAN for massive connectivity of mobile users and IoT devices", Vehicular Communications, vol. 25, p. 100250, 2020.

[33] S. Ullah et al., "UAV-enabled healthcare architecture: Issues and challenges", Future Generation Computer Systems, vol. 97, pp. 425-432, 2019. Available: 10.1016/j.future.2019.01.028.

[34] A. Mukherjee, S. Misra, A. Sukrutha and N. Raghuwanshi, "Distributed aerial processing for IoT-based edge UAV swarms in smart farming", Computer Networks, vol. 167, p. 107038, 2020.

[35] N. Nomikos, E. T. Michailidis, P. Trakadas, D. Vouyioukas, H. Karl, J. Martrat, T. Zahariadis, K. Papadopoulos, and S. Voliotis, "A UAV-based moving 5G RAN for massive connectivity of mobile users and IoT devices," Vehicular Communications, vol. 25, p. 100250, 2020.

[36] W. Feng, J. Wang, Y. Chen, X. Wang, N. Ge and J. Lu, "UAV-Aided MIMO Communications for 5G Internet of Things," in IEEE Internet of Things Journal, vol. 6, no. 2, pp. 1731-1740, 2019.

[37] F. Almalki, M. Angelides, "Propagation modelling and performance assessment of aerial platforms deployed during emergencies," 12th IEEE International Conference for Internet Technology and Secured Transactions, 2017, pp.238-243.

[38] J. Cole, F. A. Almalki, and P. R. Young, "Chipless RF Liquid Sensor," IEEE International Microwave and RF Conference (IMaRC), 2015, pp. $243-245$.

[39] Z. Ullah, F. Al-Turjman, L. Mostarda, and R. Gagliardi, "Applications of Artificial Intelligence and Machine learning in smart cities," Computer Communications, vol. 154, pp. 313-323, 2020.

[40] A. Ansari and A. A. Bakar, "A Comparative Study of Three Artificial Intelligence Techniques: Genetic Algorithm, Neural Network, and Fuzzy Logic, on Scheduling Problem," 2014 4th International Conference on Artificial Intelligence with Applications in Engineering and Technology, Kota Kinabalu, 2014, pp. 3136.

[41] A. Ashkzari and A. Azizi, "Introducing Genetic Algorithm as an Intelligent Optimization Technique", Applied Mechanics and Materials, vol. 568-570, pp. 793-797, 2014.

[42] S. Popoola, E. Adetiba, A. Atayero, N. Faruk and C. Calafate, "Optimal model for path loss predictions using feed-forward neural networks", Cogent Engineering, vol. 5, no. 1, 2018. 
International Journal of Computer Networks \& Communications (IJCNC) Vol.13, No.2, March 2021

[43] X. Li, H. Yao, J. Wang, X. Xu, C. Jiang and L. Hanzo, "A Near-Optimal UAV-Aided Radio Coverage Strategy for Dense Urban Areas," IEEE Transactions on Vehicular Technology, vol. 68, no. 9, pp. 90989109, 2019.

[44] L. Wang et al., "Communications and Networking Technologies for Intelligent Drone Cruisers," 2019 IEEE Globecom Workshops (GC Wkshps), Waikoloa, HI, USA, 2019, pp. 1-6.

[45] Z. Ullah, F. Al-Turjman and L. Mostarda, "Cognition in UAV-Aided 5G and Beyond Communications: A Survey," IEEE Transactions on Cognitive Communications \& Networking, 2020.

[46] V. Sharma, D. Jayakody and K. Srinivasan, "On the positioning likelihood of UAVs in 5G networks", Physical Communication, vol. 31, pp. 1-9, 2018.

[47] Y. Xu, L. Xiao, D. Yang, L. Cuthbert and Y. Wang, "Throughput maximization in UAV-enabled mobile relaying with multiple source nodes", Physical Communication, vol. 33, pp. 26-34, 2019.

[48] X. Liu, D. He and H. Ding, "Throughput maximization for UAV-enabled full-duplex relay system in 5G communications", Physical Communication, vol. 32, pp. 104-111, 2019.

[49] H. Dai, H. Zhang, B. Wang and L. Yang, "The multi-objective deployment optimization of UAV-mounted cache-enabled base stations", Physical Communication, vol. 34, pp. 114-120, 2019.

[50] V. Garg, Wireless Communications Networking. Estados Unidos: Morgan Kaufmann, 2007, pp. 69-135.

[51] F. A. Almalki, "Utilizing Drone for Food Quality and Safety Detection using Wireless Sensors," 2020 IEEE 3rd International Conference on Information Communication and Signal Processing (ICICSP), Shanghai, China, 2020, pp. 405-412.

[52] B. Yang, "Multiobjective Synthesis of Linear Arrays by Using an Improved Genetic Algorithm," International Journal of Antennas and Propagation, vol. 2019, pp. 1-13, 2019.

[53] F. A. Almalki "Implementation of 5G IoT Based Smart Buildings using VLAN Configuration via Cisco Packet Tracer", International Journal of Electronics Communication and Computer Engineering, 11(4), pp.56-67, 2020.

[54] "Final Evaluation Report from the Independent Evaluation Group 5G Infrastructure Association", ITU Radiocommunication Groups, 2020. [Online]. Available: https://5g-ppp.eu/wpcontent/uploads/2020/02/5G-IA-Final-Evaluation-Report-3GPP-1.pdf. [Accessed: 28- Feb- 2020].

\section{AUTHOR}

Dr Faris A. Almalki: He is an assistant professor in wireless communications and drones at Computer Engineering Dep. at Taif University, a research fellow in the Dep. of Electronic and Computer Engineering at Brunel University London. He holds a BSc in Computer Engineering from Taif University, an MSc in Broadband and Mobile Communication Networks from Kent University and a $\mathrm{PhD}$ in Wireless Communication Networks from Brunel University London. He is a Member of the IEEE Communication Society. His research interests include Low- and High-Altitude Platforms and drones along with their applications in Ad hoc wireless networks. Also, he works on research projects related IoE and AI technologies.

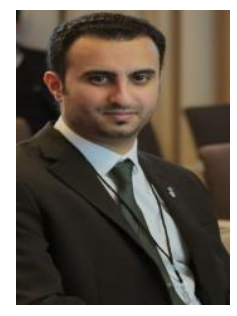

